\title{
Consejería: propuesta de un método de apoyo al paciente terminal
}

\author{
Juan P U ndurraga $\mathrm{F}^{1,2}$, Matías G onzález ${ }^{1}$, Jorge Calderón ${ }^{1}$. \\ Counseling: A comprehensive \\ method to support the terminally ill
}

During the last decades we have witnessed a progressive aging of the general population and a higher prevalence of chronic disease. This fact along with the appearance of infectious diseases like AIDS, anticipates that more patients will benefit from comprehensive palliative care. The objective of this article is to propose a simple, integral and effective method of emotional support to the patient, family and health team in palliative care (PC). PC receives little attention in medical schools, despite the great impact it has on standard health practice and quality of life of patients and their families. Our proposed method, counselling, has been empirically studied and proven to be an effective therapeutic tool in promoting behavioural changes that favour the outcome of many conditions. We believe that it facilitates PC practice, promoting direct conversation and identifying issues that can potentially cause suffering to the patients. It is based on the patient's autonomy, considers his multiple dimensions, uses and stimulates patient's own resources and coping strategies, to improve quality of life (Rev Méd Chile 2006; 134: 1448-54).

(Key words: Aging; Counseling; Palliative care)

Recibido el 30 de diciembre, 2005. Aceptado el 4 de mayo, 2006.

${ }^{1}$ Unidad de Enlace y Medicina Psicosomática, Departamento Psiquiatría, Pontificia Universidad Católica de Chile. ${ }^{2}$ Centro de Salud Familiar Madre Teresa de Calcuta, Pontificia Universidad Católica de Chile. Santiago de Chile.

«Considero que el trabajo del médico no sólo es recobrar la salud, sino disminuir el sufrimiento y el dolor; y no sólo cuando esto conduce a recuperarse, sino también cuando sirve para tener un buen pasar»

Francis Bacon

$\mathrm{D}$ urante los últimos años, hemos sido testigos de cambios demográficos importantes en la población mundial. La llamada transición epidemiológica», dada por los cambios en el estilo de vida y la mayor eficacia de los tratamientos de las principales causas de mortalidad, ha envejecido la

Correspondencia a: Dr. Juan P. Undurraga.

E mail: jpundur@puc.cl. población y ha aumentado la prevalencia de las enfermedades crónicas. Este cambio ha sido más notorio en los países desarrollados, pero se observa también en los que están en vías de desarrollo. Chile es un buen ejemplo de esto, con una esperanza de vida al nacer, en 2004, de 77,4 años ${ }^{2}$, y un incremento porcentual estimado de la población de adultos mayores (65 años o más), de 149\%, para el período comprendido entre 1990 y 
$2020^{3}$. Este envejecimiento progresivo de la población, incorpora un universo mayor de personas expuestas a patologías incurables ${ }^{4}$, que potencialmente se verían beneficiados de cuidados paliativos (CP) adecuados.

El presente artículo busca proponer un método simple, integral y efectivo, que aporte una visión general, sobre un tema de gran importancia para la práctica clínica diaria de muchos profesionales de la salud: la intervención y apoyo emocional en CP.

\section{MEDiCINA PALATIVA (MP)}

Para discutir el tema, es necesario previamente definirlo. Existen múltiples definiciones, pero seleccionamos ejemplos, que a nuestro juicio encierran conceptos importantes. «MP es el estudio y manejo de pacientes con enfermedades activas, progresivas y avanzadas, en quienes el pronóstico es limitado y en que el principal interés es la calidad de vidas, ses la atención activa y total de los pacientes y sus familias, por parte de un equipo multiprofesional, cuando la enfermedad ya no responde a tratamientos curativos y la expectativa de vida es relativamente cortas, «a medicina paliativa es un enfoque (traducción del original approach) que mejora la calidad de vida de los pacientes y familiares que enfrentan una enfermedad que amenaza la vida, a través de la prevención, evaluación y tratamiento del dolor y otros problemas físicos, psicosociales y espirituales». De estas definiciones quisiéramos destacar los conceptos de calidad de vida, como principal objetivo, la atención total y activa de los pacientes y de sus familiares, a través de la prevención, evaluación y tratamiento de las diferentes condiciones, y el enfoque multidisciplinario e integral (biopsicosocial).

Los cuidados paliativos son tan antiguos como la medicina, pero como disciplina organizada, basada en estudios empíricos, comenzó recién hace cerca de 40 años en Gran Bretaña con Cicely Saunders y el movimiento de los hospicios ${ }^{8}$. Cicely Saunders fundó el primer hospicio, St Christopher's, Sydenham, en 1967, un centro creado para dar atención a pacientes terminales, centrado en el paciente, que combina cuidado médico y de enfermería de calidad, con apoyo emocional, espiritual y social. Su labor ha generado estándares de cuidado para este tipo de pacientes en todo el mundo ${ }^{9}$.

Actualmente, existen varios modelos que dan estructura a las intervenciones para los pacientes que requieren de $\mathrm{CP}$, los que están muy influenciados por la organización y recursos del sistema de salud local, como también por las características socioeconómicas y culturales de cada lugar. Es difícil comparar el nivel de satisfacción que tienen los pacientes en relación a los diferentes modelos aplicados, dada la variedad de maneras en que esto se puede medir e interpretar. Sin embargo, se puede afirmar que la satisfacción de los pacientes $\mathrm{y}$ familiares en lugares donde funcionan modelos de atención que incorporan $\mathrm{CP}$, es alta ${ }^{8}$.

A pesar del impacto que tiene este tema en el quehacer médico y en la vida de los enfermos y su familia, los hechos nos hablan de la poca relevancia que se le otorga a algunas intervenciones y herramientas importantes en $\mathrm{CP}$, como son la comunicación efectiva y la participación activa del paciente en las decisiones médicas que le afectan. A modo de ejemplo, de acuerdo al estudio multicéntrico realizado en adultos hospitalizados graves, SUPPORT ${ }^{10}$, menos de la mitad de los pacientes hablaron con su médico del pronóstico de su condición, más de la mitad de los médicos no sabía cuáles eran los deseos del paciente en cuanto a si realizar reanimación cardiopulmonar (RCP) o no, y de los pacientes que murieron en el hospital, la mitad tuvo dolor la mayoría del tiempo (según reportes de familiares).

Es probable que la mejoría en el cuidado del enfermo terminal vaya de la mano con un entrenamiento adecuado de las personas que trabajan con este tipo de pacientes. En un estudio reciente realizado en nuestro país, en residentes de medicina de distintas especialidades de posgrado ${ }^{4}$, se evaluó la formación en CP durante los estudios de pre y posgrado y su impresión subjetiva sobre el nivel de capacitación alcanzado en relación con la atención de pacientes terminales. Los resultados no fueron muy alentadores: $76 \%$ de los residentes, independientemente del área escogida, señalaron que no tuvieron formación o cursos específicos en $\mathrm{CP}$, y del porcentaje que sí tuvo cursos específicos, la mitad consideró que su preparación era menor de $25 \%$ de lo considerado como necesario (por ellos mismos). En Estados Unidos de Norteaméri- 
ca, sólo 6 de las 126 escuelas de medicina tienen incluido, en la malla curricular, un curso sobre «muerte y cuidados al final de la vida», en el resto, es un tópico dentro de otras asignaturas ${ }^{11}$.

Otro aspecto preocupante de los CP, tiene que ver con las manifestaciones emocionales en relación a la enfermedad terminal, o su tratamiento. El estudio chileno anteriormente citado ${ }^{4}$, demostró que «as áreas relacionadas con el control de síntomas físicos tuvieron una buena evaluación (autopercibida entre los residentes), como también ocurrió con los aspectos éticos relacionados con la suspensión de terapias fútiles o dar órdenes de no resucitar (...)», sin embargo, no ocurrió lo mismo con las áreas sicológicas y antropológicas propias de la medicina paliativa, relacionadas con el sufrimiento de la persona que enfrenta una enfermedad incurable o ve vecina su muerte». Según Maguire (Cancer Research UK Phsycological Medicine Group) <el estrés y morbilidad sicológica causado por el diagnóstico de cáncer, es resuelto sólo por una minoría de los pacientes, ya que las personas que están a cargo de su cuidado tienden a evitar los aspectos emocionales. Esto sucede por dos razones fundamentales: porque no tienen las habilidades para manejar los problemas y emociones que emergen al hablar con los pacientes y familiares en profundidad y porque temen que investigar cómo se ajusta psicológicamente una persona, hará más daño que bienestar» ${ }^{2}$.

\section{MODELO DE INTERVENCIÓN}

Existen múltiples modelos de estructuración y organización de equipos de cuidado paliativo. Estos deben adaptarse a las necesidades de práctica local, a los recursos disponibles y a las prioridades establecidas por las autoridades locales y los diferentes servicios. Algunos modelos frecuentemente utilizados son ${ }^{13}$ :

1. Pacientes hospitalizados en unidades específicas de cuidados paliativos dentro del recinto hospitalario, o fuera de éste, donde pueden ser internados para manejo sintomático, 0 cuidados terminales.

2. Servicios comunitarios, compuestos por profesionales dedicados al tema de los cuidados paliativos, que brindan sus servicios en el lugar de residencia del paciente.
3. Hospitales de día, donde acuden pacientes y familiares desde sus casas para recibir cuidados adecuados.

4. Unidades especializadas en cuidados paliativos que actúan como interconsultoras dentro de un hospital general o servicio específico, que además de sus labores con pacientes y familias, ejercen una labor educativa a los profesionales en contacto con los pacientes.

Diversos grupos de estudio del área han descrito maneras de entender al paciente terminal y situaciones a las que se ven enfrentados con frecuencia, por ejemplo, el acuerdo entre familiares de no informar al paciente de su diagnóstico, y han establecido maneras más o menos estandarizadas de enfrentar estas situaciones ${ }^{13-19}$. Estos modelos requieren una base conceptual que dé sustrato al trabajo en cuidados paliativos. En ese sentido, nos parece interesante el modelo de Arranz et $\mathrm{al}^{14}$, que nos plantea una manera de entender al paciente y su entorno, de manera simple e integradora, ya que incluye, además del paciente, como el actor más importante, a sus familiares y al equipo de salud a cargo (esto último, para evitar el desgaste del equipo, con los costos que conlleva para todos los actores del proceso ${ }^{17}$ ) (Figura 1).

El modelo propone como objetivo sobtener bienestar o atenuación del sufrimiento» en estos tres protagonistas y define como sufrimiento, cuando a una persona le sacontece algo que percibe como una amenaza importante para su existencia personal u orgánica y, al mismo tiempo, siente que carece de los recursos para hacerle frente ${ }^{4}$.

Ya que el sufrimiento es una sensación subjetiva, que depende de la evaluación que el paciente haga de sus recursos, se puede afirmar que, lo más importante en la evaluación del paciente (o cualquiera de los otros actores), no es el síntoma en sí mismo, sino más bien, el grado de amenaza que éste le produce. A su vez, la evaluación de las amenazas y recursos de que dispone cada paciente, está modulado por su estado de ánimo.

De acuerdo a esto, nuestras intervenciones deben estar encaminadas en cada momento a evaluar los síntomas, situaciones y alteraciones psicológicas que el paciente perciba como amenazas e identificar, en forma paralela, los recursos 


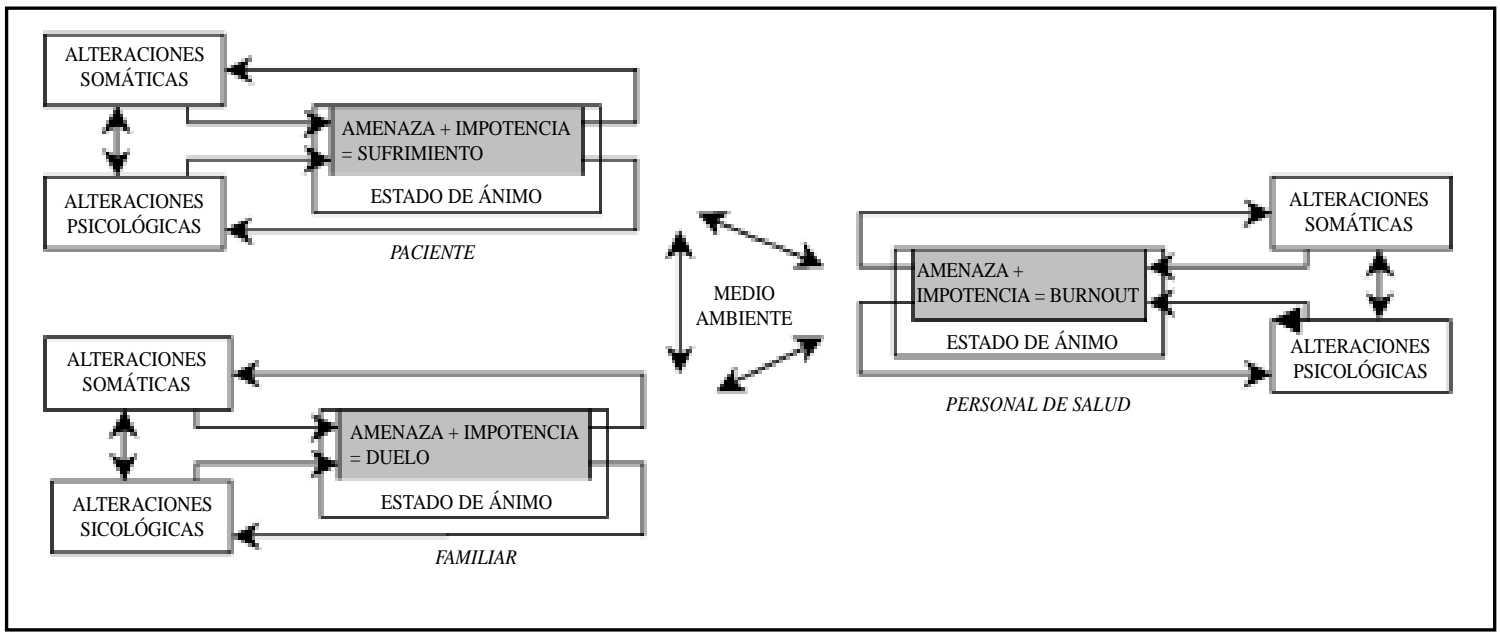

Figura 1. Modelo integral de intervención en cuidados paliativos ${ }^{12}$. Adaptado.

con que cuenta el paciente. De esta manera, podremos eliminar o atenuar los síntomas (según la prioridad que establece él mismo) y potenciar los propios recursos del paciente para enfrentarlos o mejorar su percepción de control sobre la situación y, así, disminuir el sufrimiento e incrementar su bienestar. Estaremos, además, pendientes del estado anímico del paciente para intervenir (psicológica o farmacológicamente) cuando sea necesario. En caso de que el paciente se encuentre bien adaptado a su situación, puede ser estimulado positivamente, aumentando su gama de satisfactores y disminuyendo su sensación de vulnerabilidad.

Lo descrito anteriormente se puede ampliar y aplicar en la evaluación tanto del paciente, como de los familiares, del equipo y la interacción entre ellos.

No debemos dejar de considerar también, que todo el proceso terapéutico se lleva a cabo en un ambiente con características determinadas (físicas, sicológicas y sociales), que son susceptibles a ser modificadas de acuerdo a las necesidades (y posibilidades) de cada situación.

Para aplicar este modelo, es necesario saber a ciencia cierta, qué es lo percibido como amenazante, y la única manera de saberlo es preguntarlo en forma directa al paciente. No podemos juzgar por nosotros mismos (se contrapone con la subjetividad del sufrimiento), y no podemos au- mentarle el sufrimiento al paciente a través de nuestras preguntas. En ese sentido, debemos utilizar una herramienta, que permita al paciente ser el protagonista y que a la vez, tenga un componente terapéutico.

\section{Consejería EN CUIDAdos PALIATIVOS}

La consejería (traducción del original, counseling), que comienza con Rogers, se ocupa específicamente de la exploración y resolución de la ambivalencia ${ }^{21}$. A través de una relación de aceptación, respeto y empatía, el paciente es ayudado a usar sus propios recursos para afrontar los problemas ${ }^{22}$. Posteriormente, otros autores como Carkuff, contribuyeron con otras metodologías que añaden un cierto directivismo, para reforzar a la persona del ayudado ${ }^{22}$. Dietrich ${ }^{14}$, nos da una definición más completa «en su núcleo sustancial, es esa forma de relación auxiliante, interventiva y preventiva, en la que un asesor, a través de la comunicación, intenta, en un lapso de tiempo relativamente corto, provocar en una persona desorientada o sobrecargada, un proceso activo de aprendizaje de tipo cognitivo emocional, en el curso del cual se pueden mejorar su disposición de autoayuda, su capacidad de autodirección y su competencia operativa» Se basa en el principio de autonomía, significa reconocer las 
capacidades del paciente de pensar, elegir y decidir, basándose en sus propios valores y su experiencia personal. Puede ser utilizado por cualquier profesional con la formación adecuada para ello, y no sólo por especialistas en salud mental.

Este método fue creado, inicialmente, como una manera de intervención para los pacientes con problemas de abuso de alcohol, para promover cambios de conducta y evitar confrontaciones no productivas ${ }^{21}$. Su aplicación como estrategia terapéutica, ha demostrado su efectividad para varias condiciones que involucran cambios conductuales, entre ellas, para dejar el hábito tabáqui$\mathrm{Co}^{23}$, para reducir el consumo de alcohol en grupos de riesgo y abuso $21,23,24$, para aumentar las conductas preventivas en nutrición y control de peso, ejercicio, etc ${ }^{23}$.

Creemos que la consejería es una excelente herramienta para utilizar en el tratamiento del paciente terminal, ya que permite tener una comunicación más directa y abierta, e identificar los factores que podrían producir o producen sufrimiento, utilizar los recursos de los que él dispone y promover cambios conductuales en las personas involucradas en el proceso, según sus propias necesidades, con el fin de mejorar su calidad de vida.

Según Bermejo, Carkhuff describe el proceso de consejería en fases, atendiendo a la función principal del «ayudante»y a la tarea principal del paciente 0 〈ayudado $\aleph^{2}$. Debe aclararse, que estas tres fases no son rígidas, ni tampoco están presentes necesariamente en todas las entrevistas con los pacientes.

En la primera fase, el «ayudante»debe generar un buen vínculo con el paciente, comprenderlo, acogerlo y tratar de penetrar en su punto de vista. Para esto, son destrezas fundamentales la escucha activa y la reformulación para comunicar la comprensión de lo expresado por el paciente. Por su parte, la meta del paciente sería la autoexploración de sus propias experiencias y sentimientos, tomando conciencia de las dificultades que enfrenta.

En la segunda fase, el ayudante reúne los diferentes datos que surgen, y los personaliza, de manera que el paciente los interprete, a través de la autocomprensión y reestructuración del modelo representacional. Se explora el problema o situación y sus causas o posibles incongruencias. El objetivo es que el paciente descubra dónde se encuentra, en relación a dónde quiere o necesita estar, preparándose para el cambio. Es la etapa de discernimiento, ya que se ve enfrentado a sus valores y los representados y comunicados por el ayudante.

En la última etapa, se elabora un plan de acción. El paciente analiza las diferentes alternativas existentes, delimitadas por la comprensión más profunda y realista de su situación y problemática actual. Se establecen objetivos y se identifican los recursos disponibles. Por último, se operacionalizan los pasos para lograr las metas en forma progresiva.

Este esquema básico requiere de conocimientos técnicos acerca de estrategias y variables comunicativas y, por cierto, de los temas en los que se está trabajando. También requiere de actitudes determinadas por parte del terapeuta. Uno de los pilares fundamentales es la empatía, que es la comprensión y recepción de los estados emocionales del otro, desde el punto de vista del otro. Según Bermejo, Carkhuff define empatía como da capacidad de percibir correctamente lo que expresa otra persona y de comunicar esta percepción en un lenguaje acomodado a los sentimientos de ésta $\aleph^{2}$, es decir, significa meterse en la experiencia del otro y asumir su situación, intentando abandonar las propias actitudes de defensa, y no sólo comprender, sino transmitir esta comprensión. Otras actitudes importantes, según Rogers ${ }^{14}$, son la congruencia, es decir, expresarse en forma coherente con lo que se vive y siente, y la aceptación incondicional, o sea, aceptar al otro sin enjuiciarlo. Según el mismo autor, «son estas actitudes y no los conocimientos o la habilidad del terapeuta, los principales factores determinantes del cambio terapéutico».

Respecto a las habilidades del terapeuta, la consejería pone énfasis en la comunicación asertiva, que es cuando expresamos de manera honesta $\mathrm{y}$ abierta lo que pensamos, pero manifestamos entender la posición del otro (que permite manejar nuestros sentimientos sin dejarnos conducir por ellos en el comportamiento), la escucha activa, el diálogo desde la empatía, la comunicación no verbal, el respeto a los silencios. 


\section{CONCLUSIONES}

La medicina paliativa es un área de gran importancia e impacto dentro de la medicina, cuya demanda, probablemente, irá en aumento en los próximos años, no sólo por los beneficios que trae para los pacientes y sus familiares, sino también por los cambios epidemiológicos a nivel mundial y la incorporación de nuevas condiciones como el SIDA, que hacen que haya un número creciente de personas que se beneficiarán de cuidados adecuados.

Los múltiples modelos para estructurar los equipos y las intervenciones en $\mathrm{CP}$, deben adaptarse a los recursos y características locales; sin embargo, deben siempre ser implementados de manera formal en los distintos lugares. Deben considerar al paciente, sus familiares, y a los equipos de trabajo.

El sufrimiento del paciente es una sensación subjetiva determinada por las características propias de cada persona, la evaluación de los recursos que él hace y el grado de amenaza que representan para él los diferentes síntomas 0 condiciones. Nuestras intervenciones deben estar siempre encaminadas a evaluar las amenazas y recursos del paciente, para prevenir o tratar el sufrimiento y potenciar sus recursos.

\section{REFERENCIAS}

1. Davis R, Wagner E, Groves T. Managing Chronic Disease. BMJ 1999; 318: 1090-1.

2. Ministerio de Salud de Chile. Departamento de estadísticas e información de salud. «ndicadores de estadísticas vitales, según región, año 2002». Disponible en http://deis.minsal.cl/Indicadores/ ind2004.pdf. Consultado el 8 de agosto de 2005

3. Puschel K, Araya C, Zúñiga L, Marín P. Adulto Mayor. En: Montero J, ed. Medicina Ambulatoria del Adulto. Programa de Medicina Familiar y Comunitaria. Santiago: Ediciones Universidad Católica de Chile 2001: 70-78.

4. Vial P, Ibáñez P, Umaña A, Reyes M, Viviani P, Nervi $F$ ET AL. Autoevaluación sobre Formación en Medicina Paliativa en una Cohorte de Residentes. Rev Méd Chile 2004; 132: 445-52.

5. Doyle D, Hanks G, Cherny N, Calman K. Oxford Textbook of Palliative Medicine. Oxford Universi-
La consejería es una herramienta terapéutica de efectividad probada para muchas condiciones que involucran cambios conductuales. Se basa en el principio de autonomía del paciente, que considera su multidimensionalidad y que estimula su autoeficacia, ayudándolo a descubrir sus propias estrategias adaptativas e incrementando sus recursos. Permite, además, una comunicación más directa y abierta con el paciente. Requiere de conocimientos técnicos, actitudes y habilidades específicas por parte del terapeuta. Estas características, sumadas al hecho que, con una formación adecuada, puede ser aplicado por profesionales no especialistas en salud mental, hacen de la consejería un método atractivo para ser incorporado en los programas de formación general en las escuelas de medicina.

La literatura científica nos habla de una preparación insuficiente en el área por parte de los profesionales de la salud, lo que sin duda tiene un impacto directo sobre los pacientes, los familiares, los equipos de tratamiento y el sistema de salud en general. Es un tema que requiere de mayor atención de parte de las autoridades sanitarias y académicas, que deberán, incorporar progresivamente una mayor cantidad de recursos a la formación e investigación en esta área.

ty Press. Tercera Edición, 2003.

6. López R, Nervi F, Taboada P. Manual de Medicina Paliativa. Santiago: Pontificia Universidad Católica de Chile, Facultad de Medicina, 2005.

7. World Health Organization. Palliative Care, What is it. Disponible en: http://www.who.int/hiv/ topics/palliative/care/en/index1.html. Consultado el 10 de julio de 2005.

8. Bosanquet N, Salisbury C. Providing a Palliative Care Service: Towards an evidence Base. Oxford University Press, 1999.

9. Times on Line. Dame Cicely Saunders. Disponible en: http://www.timesonline.co.uk/article/ 0,,60-1694518,00.html. Consultado el 20 de julio de 2005.

10. SUPPORT. A controlled trial to improve care for seriously ill hospitalized patients. JAMA 1995; 274: 1591-8.

11. The Lancet. Time for education in palliative care. (Editorial) 1997; 349: 1709. 
12. MAguiRe P. Barriers to psychological care of the dying. BMJ 1985; 291: 1711-3.

13. Doyle D, Woodruff R. The Internacional Association for Hospice and Palliative (IAHPC) Manual of Palliative Care. IAHPC Press, Segunda Edición. Disponible en: http://www.hospicecare.com/manual/IAHPCmanual.htm. Consultado el 27 de agosto de 2005.

14. Arranz P, Barbero J, Barreto P, Bayes R. Intervención Emocional en Cuidados Paliativos. Barcelona: Editorial Ariel, 2003.

15. Maguire P, FaulKner A. Communicate with cancer patients: 1 Handling bad news and difficult questions. BMJ 1988; 297: 907-9.

16. Maguire P, FauLKNER A. Communicate with cancer patients: 2 Handling uncertainty, collusion and denial. BMJ 1988; 297: 972-4.

17. BREWIN T. Three ways of giving bad news. Lancet 1991; 337: 1207-9.

18. FAULKNER A. ABC of Palliative Care. Communication with Patients, Families, and other Professionals. BMJ 1998; 316: 130-2.

19. FALOWFieID L, JenKINS V. Comunicating sad, bad, and difficult news in medicine. Lancet 2004; 363: 312-9.
20. Saunders C, Baines M, Dunlop $R$. Living with Dying, a Guide to Palliative Care. Oxford University Press, 1995. Tercera Edición.

21. McCAmBRiDge. Motivational interviewing is equivalent to more intensive treatment, superior to placebo, and will be tested more widely. Evid Based Ment Health 2004; 7: 52. Disponible en http://ebmh.bmijournals.com. Consultado el 20 de julio de 2005.

22. Bermejo C. Apuntes de Relación de ayuda. Centro de Humanización de la salud. Madrid: Editorial Sal Terrae, 1998.

23. LANCASTER T, OTEAD LF. Individual counselling for smoking cessation. The Cochrane Database of Systematic Reviews 2055, Issue 2. Art. N.:CD001292.pub.2 DOI10.1002/14651858.CD001292.pub2.

24. Whitlock EP, Polen MR, Green CA, Orieans CT, KIEIN J. Behavioral Counseling Interventions in Primary Care to Reduce Risky/Harmful Alcohol Use by Adults. Ann Intern Med 2004; 140: 558-69. Disponible en: http://www.ahrq.gov/clinic/ 3rduspstf/alcohol/alcomissum.htm. Consultado el 20 de julio de 2005.

Agradecimientos

Agradecemos a la Dra. Pilar Arranz, por su ayuda en la búsqueda de material bibliográfico. 